\title{
The Role of IL-10 in Gastric Spasmolytic Polypeptide-Expressing Metaplasia-Related Carcinogenesis
}

\author{
Dae Jin Park ${ }^{1}$ and Sung Eun Kim ${ }^{2}$ \\ Departments of ${ }^{1}$ Pharmacology and ${ }^{2}$ Internal Medicine, Kosin University College of Medicine, Busan, Korea
}

See "IL-10 Plays a Pivotal Role in Tamoxifen-Induced Spasmolytic Polypeptide-Expressing Metaplasia in Gastric Mucosa" by Chansu Lee, et al. on page 789, Vol. 11. No. 6, 2017

According to the GLOBOCAN 2012 report, a project of the International Agency for Research on Cancer/World Health Organization, gastric cancer is the fourth most frequently diagnosed cancer, and the third and fifth leading cause of cancerrelated mortality in men and women worldwide. ${ }^{1}$ Chronic infection with Helicobacter pylori is considered the major risk factor for gastric cancer due to inflammation of the gastric mucosa. However, the molecular mechanisms of gastric carcinogenesis remain unclear. Many attempts have sought to determine the causes of gastric carcinogenesis, especially in the early stages of gastric carcinogenesis, and multiple researches have reported that several epigenetic alterations are associated with gastric cancer, such as DNA methylation and epithelial-mesenchymal transition. ${ }^{2,3}$ Recently, spasmolytic polypeptide-expressing metaplasia (SPEM) has also been suggested to be an initiator of gastric carcinogenesis. ${ }^{4}$

In order to better understand SPEM, we need to clarify the processes related to oxyntic atrophy and gastric inflammation, which influence the development of intestinal metaplasia. ${ }^{4}$ There are two types of metaplasia that can occur in oxyntic atrophy with inflammation: intestinal metaplasia and SPEM. Both intestinal metaplasia and SPEM are related to gastric cancer progression, and therefore are regarded as precancerous states. ${ }^{4}$

SPEM has been investigated in models of acute parietal cell loss, which induces the direct conversion of chief cells into metaplastic cells. ${ }^{4}$ Tamoxifen has toxic effects on cancer cells from diverse tissues as a chemotherapeutic drug. SPEM can be induced within 3 days after oral and intraperitoneal administration of tamoxifen in a selective estrogen receptor-independent manner. Proton pump- and mitochondria-rich cells, such as osteoclasts and parietal cells, were damaged by tamoxifen, which reduced the phosphorylation efficiency of mitochondria and also affected intracellular $\mathrm{pH}$, leading to proton leaks. ${ }^{5}$ Loss of parietal cells by tamoxifen could be reversed by omeprazole, a proton pump inhibitor, suggesting its effectiveness based on active acid secretion. ${ }^{6}$ Accordingly, SPEM is considered a reversible precursor of precancerous lesions. ${ }^{7}$

Gastric mucosa returns to normal gastric histology by 3 weeks after tamoxifen is removed. The development of atrophic gastritis and metaplasia is associated with expression changes in cytokine profiles. Therefore, changes in cytokine profiles could be related to the development of SPEM. In Gut and Liver, Lee et al. ${ }^{8}$ investigated changes in cytokine profiles during the life cycle of tamoxifen-induced SPEM. They sacrificed and examined six mice in each group 3, 10, and 21 days after the administration of tamoxifen or vehicle in. At first, tamoxifen treatment induced an approximate 90\% loss of parietal cells on histology of the gastric mucosa within 3 days. Parietal cell populations returned to normal levels after wash-out periods of 10 and 21 days. Using an RNeasy Mini Kit (Qiagen, Valencia, CA, USA), gene expression assay was performed from total RNA and sequences were mapped against the mouse reference genome. Expression of interleukin (IL)-1 $\beta$, IL-12 receptor subunit $\beta 1$, tumor necrosis factor $\alpha$ (TNF- $\alpha$ ), IL-5, and IL-10 were significantly increased or decreased 3 days after tamoxifen administration despite a very low level of most cytokine and receptor RNA. The authors confirmed the changes in IL-10 expression on additional reverse transcription polymerase chain reaction (RTPCR) study. Cytokine protein levels (IL-1 $\beta$, IL-12p70, TNF- $\alpha$, IL-5, IL-10, interferon- $\gamma$, IL-4, and IL-6) were also studied using multiplex immunoassay and immunofluorescence staining. Cytokine IL-10 was particularly reduced in gastric tissues going

Correspondence to: Sung Eun Kim

Department of Internal Medicine, Kosin University College of Medicine, 262 Gamcheon-ro, Seo-gu, Busan 49267, Korea

Tel: +82-51-990-5205, Fax: +82-51-990-5055, E-mail: solefide@hanmail.net

pISSN 1976-2283 eISSN 2005-1212 https://doi.org/10.5009/gnl17395

@) This is an Open Access article distributed under the terms of the Creative Commons Attribution Non-Commercial License (http://creativecommons.org/licenses/by-nc/4.0) which permits unrestricted non-commercial use, distribution, and reproduction in any medium, provided the original work is properly cited. 
into the state of SPEM after 3 days. To localize the area between IL-10 expression and parietal cell loss/recovery, they co-stained gastric glands with specific antibodies for IL-10 (anti-IL-10) and parietal cells (anti-vascular endothelial growth factor $\beta$ ). The authors concluded that expression of IL-10 was reduced and recovered in the gastric parietal cells as shown on expression assay and immunohistochemistry. They also suggested that IL10 expression was associated with tamoxifen-induced SPEM.

IL-10 reduction and parietal cell loss were closely associated with the development of SPEM in tamoxifen-treated mice because loss of parietal cells can initiate SPEM. Inflammatory responses were stimulated by $\mathrm{IL}-10$ deficiency, leading to cancer development. ${ }^{9}$ IL-10 stimulated anticancer effects on tumorresident $\mathrm{CD}^{+}{ }^{+}$-cells in a cancer-specific immune response. ${ }^{10}$ Therefore, the anti-inflammatory and anticancer effects of IL-10 are attracting attention in the field of immunotherapy.

Lee et $a l^{8}$ revealed that IL-10 expression decreased simultaneously with SPEM development and then returned to normal levels when normal gastric histology was restored. However, they did not confirm whether decreased IL-10 expression actually caused the generation of SPEM, as tamoxifen did. Also, it remains unclear whether parietal cells are the major guardians against carcinogenesis in the gastric epithelium through their production of IL-10. In spite of these limitations, it is a reasonable inference that IL-10 could regulate the homeostasis of the gastric mucosa and inhibit the development of mucosal metaplasia, and that IL-10 production decreases could play an important role in SPEM initiation. Therefore, further investigation of the role of epithelial IL-10 in gastric tissue is needed. Continuing research on precancerous lesions in gastric molecular biology will facilitate the prevention and treatment of gastric cancers.

\section{CONFLICTS OF INTEREST}

No potential conflict of interest relevant to this article was reported.

\section{REFERENCES}

1. Ferlay J, Soerjomataram I, Dikshit R, et al. Cancer incidence and mortality worldwide: sources, methods and major patterns in GLOBOCAN 2012. Int J Cancer 2015;136:E359-E386.

2. Tan P, Yeoh KG. Genetics and molecular pathogenesis of gastric adenocarcinoma. Gastroenterology 2015;149:1153-1162.e3.

3. Asada K, Nakajima T, Shimazu T, et al. Demonstration of the usefulness of epigenetic cancer risk prediction by a multicentre prospective cohort study. Gut 2015;64:388-396.

4. Weis VG, Goldenring JR. Current understanding of SPEM and its standing in the preneoplastic process. Gastric Cancer 2009;12:189197.

5. Cardoso CM, Custódio JB, Almeida LM, Moreno AJ. Mechanisms of the deleterious effects of tamoxifen on mitochondrial respiration rate and phosphorylation efficiency. Toxicol Appl Pharmacol 2001;176:145-152.

6. Huh WJ, Khurana SS, Geahlen JH, Kohli K, Waller RA, Mills JC. Tamoxifen induces rapid, reversible atrophy, and metaplasia in mouse stomach. Gastroenterology 2012;142:21-24.e7.

7. Goldenring JR, Ray GS, Coffey RJ, et al. Reversible drug-induced oxyntic atrophy in rats. Gastroenterology 2000;118:1080-1093.

8. Lee C, Lee H, Hwang SY, Moon CM, Hong SN. IL-10 plays a pivotal role in tamoxifen-induced spasmolytic polypeptide-expressing metaplasia in gastric mucosa. Gut Liver 2017;11:789-797.

9. Neven B, Mamessier E, Bruneau J, et al. A Mendelian predisposition to B-cell lymphoma caused by IL-10R deficiency. Blood 2013;122:3713-3722.

10. Fujii S, Shimizu K, Shimizu T, Lotze MT. Interleukin-10 promotes the maintenance of antitumor CD8(+) T-cell effector function in situ. Blood 2001;98:2143-2151. 